\title{
The Right of the Children to Know Their Origin in Adopting and Medically Assisted Reproduction
}

Juelda Lamçe Ph.D.

Enarda Çuni LL.M.

European University of Tirana

\section{Doi:10.5901/mjss.2013.v4n6p605}

\section{Abstract}

The progressive developments in biotechnology present many delicate issues to be resolved in the context of family law. Among them, the child's right to know their biological origin is a controversial issue and increasingly debated in recent years, as it is in conflict with the right of the parent to remain anonymous. The question is considered of particular interest to adopted children and those born and leads through medically assisted procreation (M.A.P.) given the difference in treatment offered by the legislation in individual countries. Beyond the sociologists' reflections, the analysis is faced in legal solutions that are offered in order to reconcile two fundamental human righs, though not absolute (right to anonymity and the right to identity of the biological parents of the child). If compared to the adopted children, there is a general tendency to ensure this right effectively, towards the children born through the M.A.P. there is greater resistance. The community and international instruments provide the protection of both rights, with a trend in recent years to promote the child's right to know the truth rather than the anonymity of the biological parents. In particular, art. 8 of the European Convention on Human Rights (E.C.H.R.) while ensuring respect and protection for privacy and family rights, guarantees at the same time two opposing rights, such as the right to privacy and the protection of the personal data and the right to know one's origins. But how to reconcile these two rights given the important consequences on the right to health of the child? In addition, which option should be preferred: a) ensuring the stability of a family relationship already established, although artificial (thereby excluding any possibility to search for biological truth); or b) recognizing to the children who have reached a certain psycho-physical maturity the right to know their origins (thus sacrificing the right of the biological parent to remain anonymous)? In addition, would it be justifiable a different treatment on the point between adoption and M.A.P. ?

Keywords: right to privacy, right to identity, biological parent, adoption, medically assisted procreation.

\section{Il dibattito dottrinale sul diritto dell'individuo di conoscere le proprie origini}

II desiderio di conoscere le proprie origini è stato analizzato dai sociologi come esigenza di soddisfare tre bisogni principali dell'uomo, legati rispettivamente:

a) alla sua storia medica sulla propria salute;

b) alla proprietà (allo scopo della successione ereditaria);

c) all'esigenza psicologica di una identità, riconosciuto quale diritto fondamentale dell'uomo (O'Donovan 1988: 27).

Questo desiderio si è tradotto in un interesse legittimo a partire dagli anni 1980, con la possibilità offerta dalla biomedicina di identificare il DNA. Ci si chiede quindi, fino a che punto si estende la portata del diritto di conoscere le proprie origini? Potrebbe considerarsi sufficientemente garantito questo diritto se il figlio venisse a conoscenza del solo fatto che è nato attraverso la P.M.A. o che è stato adottato, oppure sarebbe necessario garantire al figlio anche il diritto di conoscere l'identità dei propri genitori biologici/genetici?

La dottrina distingue cinque tipologie di segreti in materia adottiva (Lenti 2004: 229).

a) il segreto dell'adottato sul fatto stesso di essere stato adottato;

b) il segreto per i terzi sul fatto che un certo bambino è stato adottato;

c) il segreto per la famiglia d'origine sulla collocazione del figlio adottato nella famiglia adottiva;

d) il segreto per l'adottato e la famiglia adottiva sulla vicenda dell'abbandono e sull'identità della famiglia d'origine (genitori, fratelli e sorelle ivi rimasti) dell'adottato;

e) il segreto per l'adottato sulla collocazione adottiva di suoi eventuali fratelli e sorelle biologici.

L'interesse dell'individuo di conoscere le proprie origini richiama il diritto al rispetto della vita privata, inteso anche come diritto di conoscere la propria storia genetica, sociale e culturale. Invero, il diritto al rispetto della propria vita privata e 
familare è garantito dagli atti internazionali nella sua accezione negativa e positiva, rispettivamente dall'art. 8 della CEDU (non può esservi ingerenza di una autorità pubblica nell'esercizio di tale diritto, a meno che ...) e dall'art. 8 della Carta di Nizza (ogni individuo ha il diritto di accedere ai dati raccolti che lo riguardano e di ottenerne la rettifica). Parte della dottrina puntualizza che la rivelazione del nome del donatore esterno alla coppia non sarebbe nè necessaria nè sufficiente a garantire al figlio la costruzione dell'autostima e di un'immagine positiva di sé: sarebbe sufficiente in questi casi conoscere caratteristiche che lo descrivono (aspetto fisico, interessi, hobby, abilità) piuttosto che conoscere il suo nome (Pennings 2001: 5).

Le tecniche di riproduzione assistita pongono tra le tante problematiche etiche e legali, anche la questione, appunto, dell'equilibrio tra l'accezione pozitiva e quella negativa della privacy. Parte di autorevole dottrina ha identificato tre criteri da prendere in considerazione per l'interpretazione e l'applicazione della legislazione in questi casi: a) la tutela della privacy, b) la responsabilità procreativa, c) l'uguaglianza (Blauwhoff 2009: 160).

a) La c.d. privacy è una nozione ampia che ricomprende non solo il diritto alla riservatezza delle informazioni personali e alla propria vita privata, ma anche la salvaguardia della libera e piena autodeterminazione della persona. In questo senso il diritto alla privacy comprende sia il diritto del figlio di chiedere e di ricevere informazioni riguardanti la propria identità, sia il diritto dei genitori naturali di rimanere anoninimi (nonchè eventualmente, il diritto dei genitori che hanno adottato il bambino di mantenere segreto il fatto della nascita del figlio attraverso la P.M.A.).

b) Sulla questione della responsabilità procreativa, oltre al legame biologico il ruolo di genitore richiede un'altro requisito fondamentale: quello di poterne assumere le proprie responsabilità (genitori incapaci legalmente quali figli nati da minorenne, da rapporti incestuosi o stupro, o attravero le P.M.A.). Può succedere anche il contrario, e cioè, che chi non ne voglia assumere le proprie responsabilità genitoriali, si veda dichiarato giudizialmente il proprio status di genitore biologico del figlio (accertamento giudiziale di paternità). In assenza quindi di una genitorialità biologica, i legislatori di molti paesi hanno provveduto ad altre forme di genitorialità legale. II problema rispetto della responsabilità genitoriale nella P.M.A., e che il riconoscimento del diritto all'educazione del figlio da parte di chi ha rinunciato in ordine a tale compito può mettere a rischio una rapporto già consolidato tra il figlio ed i suoi genitori adottivi ${ }^{1}$.

c) II criterio dell'uguaglianza richiama la questione se tutti i figli dovrebbere conoscere le proprie origini a prescindere dalle specifiche circostanze della propria nascita. Sul punto, il dibattito è tuttora apperto nella dottrina e giurisprudenza internazionale. Anche se non si tratta di un diritto assoluto, l'interpretazione della lettera dell'art. 7 della Convenzione ONU sui Diritti del Fanciullo del 1989 (il diritto del fanciullo di conoscere i propri genitori), nella parte per quanto possibile, rimane tutt'ora equivoca. Ciò in quanto il bilanciamento dei diritti coinvolti implica scelte di politica legislativa, diverse nei singoli Stati. Sulla base di quali criteri si deve stabilire se si deve garantire un diritto di conoscere oppure un diritto a non conoscere? Gli strumenti internazionali (art. 10/2 della Convenzione europea di Oveido Convenzione per la protezione dei Diritti dell'Uomo e della dignità dell'essere umano nei confronti dell'applicazioni della biologia e della medicina - e art. 5/c della Dichiarazione Universale sul Genoma Umano e i Diritti Umani) e parte della dottrina (Andorno 2004: 435)2 hanno infatti riconosciuto che in alcuni casi, il diritto di conoscere può trasformarsi in un diritto a non conoscere.

\section{Il diritto di conoscere le proprie origini nella normativa internazionale e comunitaria}

Diritto all'identità personale e diritto di conoscere le proprie origini: qual è il rapporto e il grado di tutela offerto dalla legislazione internazionale e comunitaria per ciascuna delle suddette categorie? Si ritiene che il diritto di conoscere le proprie origini costituisca un aspetto del più ampio diritto all'identità personale poichè esso può contribuire in maniera decisiva a delineare la personalità di un essere umano. L'identità personale, rivolta al passato, riguarda l'interesse alla "verità biologica" in quanto l'affermazione di un rapporto di filiazione veridico fa parte della esigenza di garantire al figlio il diritto alla propria identità. In questo senso, il contenuto del diritto all'identità personale si identificherebbe non tanto nel

\footnotetext{
${ }_{1}^{1}$ Sul diritto dei donatori di sperma di mantenere un rapporto con i bambini che hanno contribuito a creare, si veda la decisione dell'Alta Corte inglese: un donatore di sperma ha il diritto di avere incontri regolari con i suoi figli nati attraverso la fecondazione assistita, in http://www.familylawweek.co.uk/site.aspx?i=ed111506, visto il 22.05.2013.

${ }^{2} \mathrm{Ci}$ si riferisce infatti al campo della genetica e alla possibilità di prevenire certe malattie. In questi casi gli individui possono avere un interesse legittimo a non conoscere informazioni sui propri dati genetici, al fine di evitare consequenze psicologiche, puntualizzando inoltre che: a) tale interesse non possa presumersi, ma deve essere una scelta individuale; b) questo non è un diritto assoluto, nel senso che può subire restrizioni laddove la rivelazione di tale informazione è necessaria per evitare un serio rischio ai terzi.
} 
diritto ad essere sé stessi, quanto piuttosto un diritto a sapere chi si è dal punto di vista della verità biologica (RodotàTallacchini 2010). La dottrina ha parlato in proposito di una versione "forte", cioè del diritto di conoscere l'identità, e "debole", cioè del diritto di conoscere le caratteristiche genetiche, specie a fini sanitari (Ferrando 2005).

II diritto di conoscere le proprie origini è riconosciuto con alcune limitazioni negli atti internazionali e comunitari. Gli artt. 7-8 della Convenzione di New York sui Diritti del Fanciullo (20 novembre 1989) sanciscono il diritto del fanciullo di conoscere i propri genitori nella misura del possibile, adottando un concetto ampio del diritto all'identità (il diritto di mantenere i legami con i famigliari). L'art. 30 della Convenzione dell'Aia sulla Protezione dei minori e sulla cooperazione in materia di adozione internazionale (29 maggio 1993), sancisce il diritto del bambino di accedere alle informazioni riguardanti l'identità dei propri genitori nella misura consentita dalla legge dello Stato ${ }^{3}$. II nuovo testo della Convenzione europea sull'adozione dei minori del 1967 (riveduta nel 2008) garantisce un miglior equilibrio tra il diritto del minore adottato di conoscere le proprie origini, e quello dei genitori biologici di rimanere anonimi. Questo perchè l'art. 22 par. 3, sottolinea il diritto dell'adottato di conoscere le sue origini alla luce dell'art. 7 della Convenzione di New York sui Diritti del Fanciullo1989. Lo stesso tiene conto altresì del Principio n. 28 del Libro Bianco. Tale diritto non è considerato assoluto ma andrebbe bilanciato delle Autorità nazionali competenti, valutando caso per caso quando il diritto del minore dovrebbe prevalere su quello dei genitori (Bisio - Roagna 2009).

La Raccomandazione n. 1443 del 26 gennaio 2000 del Parlamento Europeo, per il rispetto dei diritti del bambino nell'adozione internazionale, invita gli Stati ad assicurare il diritto del bambino adottato di conoscere le sue origini al più tardi al compimento della maggiore età, e ad eliminare dalle proprie legislazioni nazionali ogni disposizione contraria. Anche la Risoluzione dell'Assemblea Parlamentare del Consiglio d'Europa (27 giugno 2008) riguardante l'abbandono dei neonati, invita gli Stati membri a tenere in considerazione il rispetto dei diritti dei bambini (tra cui, specificando in particolare il diritto del bambino di conoscere le sue origini) prima di definire le proprie politiche della famiglia. Tra gli scopi di tali politiche si ricordano quelli di sollecitare le madri a far conoscere la loro identità, ma soprattutto, a permettere alla madre che lo desideri di ritornare sulla sua decisione (riguardante l'anonimato del parto)4.

Infine, la Dichiarazione Universale sul Genoma Umano e i Diritti Umani riconosce uno status particolare all'informazione genetica umana considerato tra l'altro l'impatto che lo stesso ha sulla famiglia (ad esempio il rischio di malattie ereditarie o questioni legate all'aspetto culturale). L'art. 5 della stessa garantisce il diritto di decidere se essere informati o meno sui risultati di un esame genetico ${ }^{5}$.

\section{Adozione e P.M.A.: è possibile estendere il diritto di conoscere le proprie origini anche per la P.M.A.?}

Genitorialità biologica e legale non sempre coincidono: se storicamente la questione riguardava solo la paternità del figlio, oggi, lo sviluppo tecnologico ha posto la questione anche in termini di maternità del figlio. Attraverso la P.M.A. si

\footnotetext{
3 "Le autorità competenti di ciascuno Stato contraente conservano con cura le informazioni in loro possesso sulle origini del minore, in particolare quelle relative allidentità della madre e del padre ed i dati sui precedenti sanitari del minore e della sua famiglia. 2. Le medesime autorità assicurano l'accesso del minore o del suo rappresentante a tali informazioni, con l'assistenza appropriata, nella misura consentita dalla legge dello Stato".

${ }^{4}$ Si riportano di seguito i paragrafi 5, 9, 11 e 13 della Risoluzione:

5. "L'abbandono dei bambini alla nascita è una questione complessa, che coinvolge altri diritti oltre a quelli della madre: i diritti del bambino e del padre. Oggi non è più possibile ignorare i diritti del bambino, in particolare il diritto del bambino di vivere in una famiglia e di conoscere le sue origini ed è sempre difficile far passare in silenzio i diritti dei padri".

9. "L'Assemblea invita gli Stati membri:

9.1. a definire le proprie politiche della famiglia sulla base di un principio intangibile e prioritario: il rispetto dei diritti del bambino, in particolare il suo diritto di vivere nella sua famiglia e di conoscere le sue origini, diritto costitutivo dell'essere umano e vitale per il suo sviluppo";

11. "Gli Stati membri del Consiglio d'Europa devono sollecitare le madri a far conoscere la loro identità, anche se d'altra parte conviene sviluppare, in favore delle madri, modalità protette e riservate riguardanti il parto. II bambino non deve esser privato del suo diritto di conoscere le proprie origini e questo anche prima della maggiore età".

13. "L'Assemblea chiede agli Stati membri di prevedere procedure trasparenti nel caso dell'abbandono di neonati a scopo di adozione nazionale o internazionale; opportune misure debbono permettere alla madre che lo desideri di ritornare sulla sua decisione; per quanto possibile non bisogna trascurare il consenso del padre; l'adozione nazionale o internazionale non deve impedire al bambino di poter conoscere le sue origini".

${ }_{5}^{5}$ L'art. 5 sui diritti delle persone interessate stabilisce tra l'altro che: "Il diritto di ognuno di decidere di essere informato o no dei risultati di un esame genetico e delle sue conseguenze dovrebbe essere rispettato. Nella ricerca, i protocolli devono inoltre essere sottoposti a una valutazione preliminare, in conformità alle norme o direttive nazionali ed internazionale applicabili alla materia".
} 
creano, dal punto di vista giuridico, legami famigliari in cui vi è una scissione tra status e origine biologica dei soggetti interessati. Considerata la diversità delle sue forme (inseminazione e fecondazione omologa ed eterologa), si distingue non solo tra padre biologico (donatore di sperma) e padre legale, ma anche tra madre gestante e madre genetica (la quale può anche scegliere di rimanere anonima). Perciò, il principio mater semper certa, accettato sin dai tempi degli antichi romani, è ormai relativizzato dai progressi bio-tecnologici.

A un'analisi macro a livello internazionale si nota che negli Stati Uniti, quasi tutte le giurisdizioni ammettono che i figli adottivi possono accedere alle sole informazioni "non identificative" sui propri genitori biologici. Quanto invece, ai figli nati attraverso le P.M.A., alcune corti hanno consentito l'accesso alle informazioni sull'identità dei donatore di sperma che fosse garantito precedentemente l'anonimato, per poter risolvere questioni legate alle malattie ereditarie (Sutherland 2012: 444). Nell'Africa del Sud, i figli nati attraverso le P.M.A. hanno diritto di accedere alle sole informazioni "non identificative" sui propri genitori "genetici". Al contrario, i figli adottivi possono accedere all'identità dei propri genitori una volta raggiunta la maggiore età, oppure se minori, quando vi sia un ordine del giudice che valuti l'interesse superiore dei figli (Sutherland 2012: 406).

Nel quadro europeo, rispetto all'adozione e i contatti tra adottato e famiglia di origine, in alcuni Stati l'adottato non può accedere a informazioni sull'identità del genitore biologico (Austria, Principato di Monaco, Bulgaria, Russia, Macedonia); in altri, il diritto di conoscere la propria origine è parzialmente garantito a partire da una certa età (Germania, Croazia, Ungheria, Lettonia, Portogallo)6: in altri ancora, tale diritto è soggetto a valutazioni e autorizzazioni da parte dei giudici (Bulgaria, Estonia, Lituania, Svizzera, Spagna, Regno Unito e Irlanda). In alcuni casi, vi è una discrepanza tra ciò che la legge garantisce e il riflesso sociologico di tali scelte (Labos 2007: 1760). La legge italiana riconosce alla madre la facoltà di non essere nominata nell'atto di nascita, c.d. "parto segreto"7. Tale scelta del legislatore italiano avrebbe come fine quello di evitare situazioni di abbandono dei figli non desiderati. La legge francese al contrario, con una disciplina più articolata di quella italiana, prevede il diritto dell'adottato di conoscere i propri genitori d'origine. La scelta della madre di rimanere anonima non è irreversibile come in Italia: se richiesto dal figlio, attraverso una figura intermedia (il Consiglio Nazionale per l'Accesso alle Origini Personali istituito con decreto del 3 maggio 2002) è possibile ottenere il consenso della madre (Ferrando 2005: 11).

Rispetto ai figli nati attaverso la P.M.A., la Germania è classificata tra i primi paesi che hanno garantito il diritto del figlio di conoscere le proprie origini, seguita dai Paesi Bassi la cui legislazione prevede la registrazione dei donatori di gameti. La legge portoghese del 2007, al contrario, non consente ai concepiti attraverso la donazione di gameti di conoscere le proprie origini (Blauwhoff 2009). In Italia l'attuale legge sulla procreazione assistita (Legge n. 40 del 19 febbraio 2004) adotta una posizione restrittiva, vietando esplicitamente l'inseminazione eterologa, comminando perfino una pena nel caso della sua adozione. Sul trattamento differenziato tra figli adottati e figli nati da fecondazione eterologa, si è da tempo evidenziata la necessità di una legge che disciplini anche questa seconda ipotesi. Se la legge sull'adozione prevede che l'adottato che abbia raggiunto i venticinque anni può conoscere l'identità dei propri genitori biologici, a prescindere da motivi di carattere sanitario (art. 28 legge n. 184/1983), la legislazione attuale non consente la fecondazione eterologa mettendo in discussione la disparità di trattamento nei due casi. II Comitato Nazionale per la Bioetica (C.N.B.) raccomanda che i genitori rivelino al figlio le modalità del suo concepimento attraverso filtri e criteri appropriati e raccomanda altresì che al nato si riconosca sempre il diritto di accedere a quei registri dove sono conservati i dati genetici e la storia clinica dei datori di gameti, dato che trattasi di notizie a volte indispensabili per la sua salute. Per quanto riguarda invece il diritto del nato di conoscere anche i dati anagrafici del donatore/donatrice di gameti, il Comitato non è stato in grado di dare una risposta univoca8.

Questo quadro di soluzioni eterogenee rifletterebbe le diversità di disciplina tra adozione e P.M.A. Si nota innanzittutto il diverso momento dell'assunzione di responsabilità da parte dei genitori legali: se nell'adozione questo avviene successivamente alla formazione del feto, nel P.M.A. ciò avviene ancora prima. Inoltre, se l'adozione è un istituto incentrato sull'interesse del minore, la P.M.A. è una tecnica che pone al centro del suo obiettivo i futuri genitori e cerca di offrire una soluzione all'infertilità della coppia o del singolo. Sul punto, non pare condivisibile la concezione di chi vede nell'eterologa, un modo per mascherare al gruppo sociale e famigliare di appartenenza il problema della sterilità,

\footnotetext{
${ }^{6}$ Corte Europea dei Diritti dell'Uomo Godelli c. Italia, 25 settembre 2012, ric. n. 33783/09, par. 31.

7 La legge n. 149 del 2001 Legge 28 marzo 2001, n. 149 "Modifiche alla legge 4 maggio 1983, n. 184, recante "Disciplina dell'adozione e dell'affidamento dei minori" garantisce il diritto dell'adottato alla conoscenza delle proprie origini, tranne per il caso in cui il l'adottato non sia riconosciuto dalla madre natural e o quando anche uno solo dei genitori biologici abbia dichiarato di non voler essere nominato (art. 28).

${ }^{8}$ Parere del C.N.B., Conoscere le proprie origini biologiche nella Procreazione Medicalmente Assistita eterologa, del 25 novembre 2011.
} 
dando alla coppia una parvenza di fecondità (Nicolussi 2012:7). Invero, senza poter trattare in questa sede la questione dell'ammissibilità dell'eterologa, si sottolinea che l'obiettivo della legge è quello di offrire soluzioni a interessi meritevoli di tutela, tra cui certamente quello di avere un figlio biologico (per quanto ciò sia possibile). Sicuramente la soluzione dei problemi che derivano dalla c.d. split fatherhood o split motherhood, non può essere individuata in un divieto generico alla pratica dell'eterologa, ma nello stabilire regole precise che garantiscano effettivamente l'equilibrio degli interessi coinvolti. Infine, c'è chi agomenta che la P.M.A. (senza distinguere tra omologa ed eterologa) dovrebbe essere preferita rispetto all'adozione poichè nella prima vi sarebbe un legame più forte tra genitori e figli a causa del rapporto genetico esistente tra loro (Storrow 2006: 483).

Tuttavia, le diversità delle pratiche analizzate non sembrano di poter giustificare un diverso trattamento rispetto al diritto del figlio di conoscere le proprie origini (generalmente riconosciuto nel primo e negato nel secondo).

\section{La giurisprudenza di Strasburgo}

A partire dal 1989, (il caso Gaskin)9 ${ }^{9}$ la Corte ha dichiarato, che l'interesse del ricorrente di conoscere le proprie origini è un'interesse primordiale protetto dalla Convenzione (di cui i figli necessitano per conoscere e comprendere la loro infanzia), e che non può ritenersi venuto meno per il solo fatto dell'età avanzata. In questo caso la ricorrente non poteva ottenere informazioni circa l'identità di sua madre biologica, poichè la legge nazionale (francese) le consentiva di rimanere anonima. La Corte in quella occasione decise che non vi era stata violazione dell'art. 8 della CEDU e che la Francia non aveva oltrepassato il suo margine di apprezzamento nel garantire tale diritto. Infatti, la legislazione francese aveva previsto l'istituzione del Consiglio Nazionale per l'Accesso all'Informazione sull'Origine Personale, tramite il quale la ricorrente poteva chiedere lo svelamento dell'identità della madre, previa autorizzazione della stessa.

Nel caso Odièvre ${ }^{10}$, la Corte, chiamata a valutare le norme sull'anonimato dopo il parto, ha stabilito che l'interesse del figlio di conoscere le sue origini è riconosciuto nell'ambito del rispetto della vita privata e non della vita famigliare: infatti, l'accesso all'informazione non mette in discussione il rapporto tra l'adottato e i genitori adottivi, ma ha come scopo quello di scoprire certi fatti legati alla vita privata e all'identità personale dell'individuo, quali la nascita, l'abbandono, ecc. (Roagna 2012: 53). In questo caso, la Corte ha riconosciuto agli Stati membri una certa discrezionalità nella valutazione delle misure che garantiscono il diritto ex art. $8 \mathrm{CEDU}$, ma ha precisato anche che questo margine di discrezionalità deve garantire un giusto equilibrio degli interessi in giocco.

La decisione del caso Gaskin viene ripresa dalla Corte di Strasburgo anche nel caso Jäggi contro Svizzera ${ }^{11}$, riguardante la richiesta di prelievo di campioni di DNA dalla salma di un uomo nell'ambito di un'azione di accertamento di paternità (il defunto si era in vita dichiarato contrario a sottoporsi a test genetici). Secondo la Corte non vi era violazione del diritto del presunto padre (o meglio della sua salma) al rispetto della vita privata, e quindi tale diritto cesserebbe al termine della vita medesima. Dall'analisi delle due sentenze si potrebbe cogliere il diverso raggionamento della Corte: se nel caso Jäggi, la volontà espressa dal presunto padre biologico, non coercibile in vita, può essere contravvenuta dopo la morte, nel caso Mikulic la protezione dei terzi preclude la sottoposizione coatta ad analisi mediche incluso il test del DNA per l'accertamento di paternità (Campiglio 2007: 395).

La questione se il minore nato mediante la tecnica dell'inseminazione artificiale vedesse tutelato meglio il proprio interesse mediante l'anonimato del genitore biologico, oppure mediante il diritto di conoscere l'identità di quest'ultimo fu affrontata dalla Corte di Strasburgo nel caso Godelli contro Italia ${ }^{12}$. Al contrario, secondo la Corte Costituzionale italiana l'anonimato della madre è lo strumento adottato dalla legge nazionale proprio per garantire i diritti del bambino (che altrimenti sarebbero compromessi dalla decisione della madre di abbortire 0 abbandonare un figlio non desiderato).

Infine, rispetto ai genitori transessuali la giurisprudenza di Strasburgo ha stabilito che il divieto nei confronti di una persona transessuale di essere registrato come il padre del figlio nato attraverso l'inseminazione artificiale, non costituisce violazione dell'art. 8 CEDU ${ }^{13}$. La Corte precisa infatti che, in mancanza di un standard comune tra i paesi del Consiglio con riguardo ai diritti parentali degli individui transessuali, deve essere garantito alle autorità nazionali un ampio margine di discrezionalità.

\footnotetext{
${ }^{9}$ Corte Europea dei Diritti dell'Uomo, Gaskin c. Regno Unito, ricorso n. 10454/83, sentenza del 7 Iuglio 1989.

${ }^{10}$ Corte Europea dei Diritti dell'Uomo, Odièvre c. Francia, ricorso n.42326/98, sentenza del 13 febbraio 2003.

${ }^{11}$ Corte Europea dei Diritti dell'Uomo, Jäggi contro Svizzera, ricorso n. 58757/00, sentenza del 13 luglio 2006.

${ }^{12}$ Corte Europea dei Diritti dell'Uomo, Godelli c. Italia, ricorso n. 33783/09, sentenza del 25 settembre 2012.

${ }^{13}$ Corte Europea dei Diritti dell'Uomo, X, Y, Z c. Regno Unito, ricorso n. 21830/93, sentenza del 22 aprile 1997.
} 


\section{Considerazioni conclusive}

Alla luce delle considerazioni svolte, relative al riconoscimento e attuazione del diritto del figlio di conoscere le sue origini, si possono sintetizzare le seguenti riflessioni.

1. Rispetto alla ricerca dell'equilibrio tra i due diritti coinvolti della privacy, il diritto del figlio di conoscere le sue origini sta ottenendo un riconoscimento sempre più ampio sia nelle politiche legislative raccomandate dal Parlamento europeo, sia nella giurisprudenza della Corte di Strasburgo. Nello specifico, le prime sollecitano gli Stati membri (ormai da alcuni anni), di favorire politiche legislative che incentivino le madri a far conoscere la loro identità, ma soprattutto, che permettano loro di riconsiderare la loro decisione (riguardante l'anonimato del parto).

2. Quanto alla responsabilità procreativa, le considerazioni svolte in questa sede, nonchè nello specifico, la decisione dell'Alta Corte inglese (sul diritto del donatore di sperma di avere incontri regolari con i suoi figli nati dalla fecondazione eterologa), fanno riflettere sull'opportunità di stabilire regole precise in tema di diritti e doveri dei genitori verso i figli. Al contrario, la soluzione dei problemi che derivano dalla c.d. split fatherhood o split motherhood, non può essere individuata in un divieto generico alla pratica dell'eterologa.

3. Infine, con riferimento al criterio dell'uguaglianza rispetto al diritto del figlio di conoscere le sue origini, il trattamento differenziato tra figli adottivi e figli nati tramite P.M.A. non può trovare giustificazione sulla base delle diversità evidenziate tra le due pratiche. In realtà, anche se tale diritto non è considerato un diritto assoluto (ma un diritto che va garantito dai singoli Stati nella misura del possibile), l'esigenza psicologica di una identità è riconosciuta quale diritto fondamentale dell'uomo: questo a prescindere dal fatto che si tratti di adozione o di P.M.A. Ciò vuol dire che, nei casi in cui si deve trovare un equilibrio tra diritti fondamentali, la scelta non può essere quella del sacrificio dell'uno a favore dell'altro (i diritti degli adulti su quelli dei minori).

Per questi motivi, in termini di politica legislativa si dovrebbe tentare la soluzione che incentiva il non anonimato nelle pratiche della P.M.A. Se ciò non dovesse risultare praticabile, si potrebbe ipotizzare una garanzia minimalista $\mathrm{e}$ sussidaria rispetto alla prima: l'estensione della soluzione francese, riguardante la revocabilità dell'anonimato da parte della madre biologica nel parto anonimo, anche ai casi di P.M.A. Non si potrebbe infatti negare al figlio, quale soggetto che non ha scelto di venire al mondo, il diritto di conoscere le sue origini biologiche (considerate le implicazioni che tale divieto pottrebbe comportare sulla sua salute fisica e psicologica). Del resto, la tendenza negli ultimi anni in quasi tutto il mondo è nel senso di favorire quest'ultima opzione sia nelle scelte legislative che in quelle giurisprudenziali.

\section{Bibliografia}

Andorno R., (2004) The right not to know: an autonomy based approach, in Law, ethics and medicine, 30: 435-439.

Bisio A., Roagna I., (2009) L'adozione internazionale di minori: normativa interna e giurisprudenza europea, Milano.

Blauwhoff J. R., (2009) Foundational facts, relative truths: a comparative law study on children's right to know their genetic origins, United States.

Campiglio C., (2007) Con la morte, l'uomo perde il diritto al rispetto della vita privata, in Diritti umani e Diritto internazionale: 394-397.

Ferrando G., Gli interventi della Corte Europea dei Diritti dell'Uomo in materia di famiglia e il loro rilievo per la disciplina interna: gli artt. 8 e 12 della Convenzione, nella Conferenza La famiglia nel diritto internazionale privato, nel diritto comunitario e nella giurisprudenza della Corte Europea dei Diritti dell'Uomo CSM, Roma 25 gennaio 2005.

Labos A. at al., (2007) Legislated right for donor-insemination children to know their genetic origin: a study of parental thinking, Human Reproduction, 22: 1759-1768.

Lenti L., (2004) Adozione e segreti, in Nuova giurisprudenza civile e commerciale, II: 229-253.

Nicolussi A., (2012) Fecondazione eterologa e diritto di conoscere le proprie origini. Per un'analisi giuridica di una possibilità tecnica, in Associazione Italiana dei Costituzionalisti, 1: 1-18.

O'Donovan K., (1988) A Right to Know One's Parentage?, in International Journal of Law and the Family, 2: 27-45.

Pennings G., (2001) The right to privacy and access to information about one's genetic origins, in Med Law 20 (1): 1-15.

Roagna I., (2012) .Protecting the right to respect for private and family life under the European Convention on Human Rights, Council of Europe human rights handbooks, Strasbourg.

Rodotà S., Tallacchini M., (2010) Ambito e fonti del biodiritto, in Trattato di biodiritto, diretto da S. Rodotà e P. Zatti, vol. I, Giuffrè, Milano.

Storrow R. F., (2006) Marginalizing adoption through the regulation of assisted reproduction, Capital University Law Review, 35 (2): 479-516.

Sutherland E. E., (2012) The Future of Child and Family Law, New York. 